\title{
Expression and function of Pdcd4 in mouse endometrium during early pregnancy
}

\author{
Yue Zhang ${ }^{1, *}$, Mingyun Ni ${ }^{1, *}, \mathrm{Na} \mathrm{Liu}^{1}$, Yongjiang Zhou ${ }^{2}$, Xuemei Chen ${ }^{1}$, Yubin Ding ${ }^{1}$, \\ Junlin $\mathrm{He}^{1}$, Yingxiong Wang ${ }^{1}$, Xueqing Liur, Yanqing Geng ${ }^{1}$ and Liling Xie ${ }^{3}$ \\ ${ }^{1}$ Laboratory of Reproductive Biology, School of Public Health and Management, Chongqing Medical University, \\ Chongqing, People's Republic of China, ${ }^{2}$ Maternal, Child and Adolescent Health, School of Public Health, Hainan \\ Medical College, Hainan, People's Republic of China and ${ }^{3}$ The First Affiliated Hospital of Chongqing Medical \\ University, Chongqing, People's Republic of China
}

Correspondence should be addressed to Y Geng or L Xie; Email: aqing000@163.com or 582570124@qq.com

*(Y Zhang and M Ni contributed equally to this work)

\begin{abstract}
Embryo implantation is a complex process involving synchronised crosstalk between a receptive endometrium and functional blastocysts. Apoptosis plays an important role in this process as well as in the maintenance of pregnancy. In this study, we analysed the expression pattern of programmed cell death $4(\mathrm{Pdcd} 4)$, a gene associated with apoptosis in the mouse endometrium, during early pregnancy and pseudopregnancy by real-time quantitative polymerase chain reaction, in situ hybridisation, Western blotting and immunohistochemistry. The results showed that Pdcd4 was increased along with days of pregnancy and significantly reduced at implantation sites (IS) from day 5 of pregnancy (D5). The level of Pdcd4 at IS was substantially lower than that at interimplantation sites (IIS) on D6 and D7. In addition, Pdcd4 expression in the endometrium was reduced in response to artificially induced decidualisation in vivo and in vitro. Downregulation of Pdcd4 gene expression in cultured primary stromal cells promoted decidualisation, while upregulation inhibited the decidualisation process by increasing apoptosis. These results demonstrate that $\boldsymbol{P d c d} 4$ is involved in stromal cell decidualisation by mediating apoptosis and therefore plays a role in embryo implantation in mice. Reproduction (2018) 155 393-402
\end{abstract}

\section{Introduction}

Embryo implantation is a multifactorial and complex event that results in the establishment of a successful pregnancy. During this process, a well-designed molecular dialogue between a potential embryo and the maternal endometrium is necessary to complete implantation through positioning, adhesion and invasion. These independent but inseparable events involve several specific regulatory factors, such as hormones, cytokines and the expression of corresponding receptors and co-receptors (Paria et al. 1993, Bowen \& Burghardt 2000, Dey et al. 2004). When the blastocyst adheres to the endometrial epithelium, stromal cells near the blastocyst begin to proliferate and differentiate into decidual cells like 'fertile soil' to aid successful embryo implantation. The establishment and maintenance of normal decidua play vital roles in embryo implantation and development. Any errors in these processes can result in a failed pregnancy (Huet-Hudson et al. 1990, Bigsby \& Li 1994, Das et al. 1997, Everett et al. 1997, Carson 2000).
According to a WHO comparative report, at least one in four couples suffers from primary or secondary infertility in developing countries; in developed countries, the incidence of infertility is approximately $15 \%$ and has remained stable since the turn of the twentieth century (Messinis et al. 2016). In failed human pregnancies, an estimated $30 \%$ of conceptions are lost following implantation in the third or fourth week of gestation (Larsen et al. 2013). Although a variety of assisted reproductive technologies have been developed, and medical technology has been improved, the rate of successful pregnancy has not obviously increased. Therefore, exploring the factors and mechanisms contributing to failed pregnancies is important for treating infertility and other related diseases.

Many studies have explored the roles of proliferation and differentiation during the processes of decidualisation and implantation; however, the role of apoptosis and related molecular events remains unclear. In recent years, apoptosis has attracted more and more attention by scholars. For example, the cytokine receptor 
syndecan1 (SDC1) and its ligands, which are known to play a role in the apoptosis of tumour cells, are also involved in the implantation process (Boeddeker \& Hess 2015). However, there are no reports on whether the programmed cell death $4(\mathrm{Pdcd} 4)$ is related to embryo implantation.

PDCD4 is a highly conserved protein with two basic domains at the N/C-terminus and is known to inhibit carcinogenesis, tumour progression and invasion by preventing gene transcription and translation (Zhang et al. 2016). PDCD4 can also regulate several biological function via several factors and pathways, such as S6K1, microRNA-21 (miR-21), mTOR and pAkt (LankatButtgereit \& Goke 2009). In addition, PDCD4 was shown to be downregulated in several cancers, such as thyroid adenoma, colon carcinoma, oesophageal cancer and ovarian cancer (Avi Ashkenazi 1998, Green \& Reed 1998, Mudduluru et al. 2007, Ding et al. 2016). In hydatidiform mole tissues and choriocarcinoma cells, PDCD4 was negatively regulated by overexpression of miR-21 and promoted proliferation, migration and invasion (Wang et al. 2017).

This study aimed to explore the expression pattern and function of the $P d c d 4$ gene in mouse endometrium during early pregnancy and pseudopregnancy. Our findings also clarified the role of $P d c d 4$ in decidualisation and helped elucidate the molecular mechanisms involved in implantation.

\section{Materials and methods \\ Ethical approval and reagents}

Mice (Mus musculus) were obtained from the Laboratory Animal Center of Chongqing Medical University (Chongqing, China (Certificate: SICXK (YU) 2007-0001)). All animals/ human procedures were approved by the Ethical Committee of Chongqing Medical University, and informed consent was obtained from all human subjects.

Major reagents: All primers were synthesised by Songon Biotech Co., Ltd. (Shanghai, China). RNAiso Plus Reagent (D9108B), SYBR Green and a reverse transcription kit (DRR047A) were purchased from TaKaRa. The oligonucleotide probe for the Pdcd4 gene labelled with digoxigenin was designed and synthesised by Dingguo BioTechnology Co., Ltd. (Beijing, China), and the oligonucleotide sequence was as follows: 5'-GACACTGAATGTGAACCCCACTGACCCT-3'. The in situ hybridisation kit was purchased from the same company. The anti-PDCD4 (D29C6), anti- $\beta$-actin (\#3700), anti-cleaved caspase3 (\#9664) and anti-caspase3 (\#9662) antibodies used in Western blotting (WB) were purchased from Cell Signaling Technology. The anti-BAX (ab32503) and anti-BCL2 (ab32124) antibodies were purchased from Abcam. The anti-PDCD4 (ab79405) antibody used in immunohistochemistry (IHC) was also purchased from Abcam. A diaminobenzidine kit (DAB ZL-9018) was used.

\section{Animals and tissue collection}

Adult virgin female and male Kunming mice (20-25 g, 8 weeks old) were housed in a specific pathogen-free animal room under a controlled photoperiod ( $12 \mathrm{~h}$ light/12 h darkness) at $22 \pm 2{ }^{\circ} \mathrm{C}$ and $55 \pm 10 \%$ relative humidity. All mice had access to water and food ad libitum. Female mice were mated with the fertile males or vasectomised males at a ratio of 3:1 to induce pregnancy and pseudopregnancy, and the day of appearance of a vaginal plug was considered day 1 of pregnancy (D1) or day 1 of pseudopregnancy (PD1). The pregnant and pseudopregnant mice were randomly assigned to several groups (D1, D4-D7 and PD1-PD7), with 8 mice per group. The implantation sites (IS) on D5 tissues were identified by intravenous injection of trypan blue through the tail vein. The mice were killed between 08:00 and 09:00 h, and part of the endometrium was collected and stored in liquid nitrogen for real-time quantitative polymerase chain reaction (RT-qPCR), WB and in situ hybridisation (ISH); the remaining sample was fixed in $4 \%$ paraformaldehyde for immunohistochemistry (IHC) (Ding et al. 2012).

An established procedure for artificially inducing decidualisation (ID) in vivo was adopted from a previous publication (Long et al. 2015). Briefly, one uterine horn from each PD4 mouse was injected with $10 \mu \mathrm{L}$ corn oil, while the other uterine horn did not receive any treatment and served as a negative control (Non-ID). The mice were killed by cervical dislocation four days later. After the wet weight of the uterine tissues was measured, part of the uterine tissue was stored in liquid nitrogen for RT-qPCR and WB, and the rest was fixed in $4 \%$ paraformaldehyde for IHC.

All human samples were obtained from pregnant women visiting the clinic at the Department of Gynecology in the First Affiliated Hospital of Chongqing Medical University from August to October $2014(n=16)$. The samples were obtained from the control group (the patients underwent induced abortion surgery without any signs of pregnancy disorders, $n=8)$, threatened-abortion patients $(n=5)$ and missedabortion patients $(n=3)$ by surgical operation (at 6-9 weeks of pregnancy). There were no significant differences in age, weight and the gestational weeks among the participants. (The inclusion criteria used to select human participants for this study were as follows: (1) Positive pregnancy test; (2) Regular menstrual cycle; (3) No history of genetic disease; (4) No sexually transmitted disease; (5) Non-immunodeficient; (6) No endocrine disease; (7) No chromosomal abnormalities; (8) Normal uterine anatomy (when examined by ultrasound); (9) Embryo sizes consistent with gestational age.).

\section{Real-time quantitative polymerase chain reaction}

Total RNA was extracted from endometrial tissues and cells using RNAiso Plus Reagent according to the manufacturer's protocol. The cDNA was synthesised from an appropriate quantity of total RNA treated with DNase I in a $10 \mu \mathrm{L}$ reaction system using an RT-qPCR kit. $\beta$-actin, a housekeeping gene, was used to normalise the data (Kang et al. 2002, Ozpolat et al. 2007). The specific primers used for the Pdcd4, Dtprp (decidual/trophoblast prolactin-related protein, a marker for 
Table 1 Primer sequences for qPCR.

\begin{tabular}{|c|c|c|}
\hline Genes & & Sequence of primers $5^{\prime}-3^{\prime}$ \\
\hline \multirow[t]{2}{*}{$\mathrm{Pdcd} 4$} & Forward & ACTGACССТGACAATTTAAGCG \\
\hline & Reverse & TTTTCCGCAGTCGTCTTTTGG \\
\hline \multirow[t]{2}{*}{ Dtprp } & Forward & AGCCAGAAATCACTGCСАСТ \\
\hline & Reverse & TGATCCATGCACCCATAAAA \\
\hline \multirow[t]{2}{*}{$\beta$-actin } & Forward & TGGAATCCTGTGGCATCCATGAAAC \\
\hline & Reverse & TAAAACGCAGCTCAGTAACAGTCCG \\
\hline
\end{tabular}

in vitro decidualisation) (Liang et al. 2010) and $\beta$-actin genes are shown in Table 1. Transcripts were quantified using SYBR Green, and the PCR analyses were performed on a Bio-Rad CFX Manager 3.1 Detection System (USA). The $2^{-\Delta \Delta C T}$ method was used to calculate relative expression levels of $\mathrm{Pdcd} 4$ in the endometrium on different days of pregnancy for different model systems.

\section{Western blotting}

Proteins were extracted from the endometrial tissues and cells by using lysis buffer. Protein concentrations were determined with a bicinchoninic acid protein assay kit (Beyotime Institute of Biotechnology, Jiangsu, China) according to the manufacturer's protocol. Samples were boiled in $5 \times$ sodium dodecyl sulphate (SDS) sample loading buffer for $10 \mathrm{~min}$ and loaded onto a $10 \%$ SDS-PAGE gel. Samples were electrophoresed for $100 \mathrm{~min}$ and were then transferred onto PVDF membranes. Membranes were blocked for $1 \mathrm{~h}$ at room temperature in PBST containing 5\% skim milk or BSA powder. Membranes were incubated with the following antibodies overnight at $4^{\circ} \mathrm{C}$ : anti-PDCD4 (1:1000 dilution), anti-Cleaved Caspase3 (1:1000 dilution), anti-Caspase3 (1:1000 dilution), anti-BAX (1:1000 dilution), anti-BCL2 (1:500 dilution) and anti- $\beta$-actin (1:2000 dilution) and washed three times (for $15 \mathrm{~min}$ each) with PBST. The washing processes were repeated after incubation for $1 \mathrm{~h}$ with the goat anti-rabbit IgG or goat anti-mouse $\operatorname{lgG}$. The protein expression was detected by ECL Plus reagent according to the manufacturer's protocol and analysed using Quantity One 4.6 software.

\section{In situ hybridisation}

According to a previous report (Ni et al. 2002), uteri were cut into pieces and flash frozen in liquid nitrogen. Frozen sections $(10 \mu \mathrm{m})$ were mounted continuously and fixed in $4 \%$ paraformaldehyde solution in PBS for $1 \mathrm{~h}$. After three washes with PBS, the tissues were treated with Triton (1\%) for $20 \mathrm{~min}$. After three washes with PBS, the sections were prehybridised in a hybridisation buffer at $37^{\circ} \mathrm{C}$ for $2-3 \mathrm{~h}$ and then hybridised in hybridisation buffer with DIG-labelled antisense or sense RNA probe $(1-5 \mathrm{mg} / \mathrm{mL})$ at $55^{\circ} \mathrm{C}$ for $16 \mathrm{~h}$. After hybridisation, the sections were washed in $5 \times$ SSC, $2 \times$ SSC, $0.5 \times$ SSC and $0.2 \times$ SSC twice for $10 \mathrm{~min}$ each. Nonspecific binding in the sections was blocked with $0.5 \%$ block mix, and then, the sections were incubated with goat anti-DIG antibody. The final signal was visualised with 5-bromo-4-chloro-3-indolyl phosphate and nitroblue tetrazolium. The sections were counterstained with methyl green. The sections treated with the DIG-labelled sense probe were negative. The images were captured by using an Olympus microscope (BX40, Olympus).

\section{Immunohistochemistry}

Tissue samples were embedded in paraffin according to standard histological procedures and sectioned to obtain continuous sections of $4 \mu \mathrm{m}$ thickness. The sections were deparaffinised in xylene and rehydrated in descending concentrations of ethanol after antigen retrieval in sodium citrate buffer and cooled down to room temperature. The tissue sections were blocked in 10\% normal goat serum for $30 \mathrm{~min}$ and incubated with anti-PDCD4 antibody (1:300 dilution) at $4^{\circ} \mathrm{C}$ overnight. Then, the tissue sections were incubated with a secondary antibody (goat anti-rabbit $\operatorname{lgG}$ ) at $37^{\circ} \mathrm{C}$ for $30 \mathrm{~min}$. Next, the sections were incubated with streptavidinconjugated horseradish peroxidase at $37^{\circ} \mathrm{C}$ for $30 \mathrm{~min}$. The staining was performed by using DAB substrate for $5 \mathrm{~min}$ at room temperature and terminated by rinsing with water. The sections were subsequently stained with haematoxylin. The images were captured by using an Olympus microscope (BX40, Olympus).

\section{Isolation of endometrial stromal cells (ESCs) and in vitro decidualisation}

Mouse uterine stromal cells were isolated as previously described (Osteen et al. 1989). The uteri obtained from PD4 mice were washed thoroughly with D-Hanks solution. In addition, they were cut into small fragments with sterile scissors. The uterine tissues were placed in an appropriate amount of HBSS containing 1\%wt/vol trypsin and $6 \mathrm{mg} /$ $\mathrm{mL}$ dispase at $4^{\circ} \mathrm{C}$ for $2 \mathrm{~h}$, and then, they were incubated at $37^{\circ} \mathrm{C}$ for $30 \mathrm{~min}$. The digested uteri were gently agitated by pipetting to dislodge sheets of luminal epithelial cells. After centrifugation, the supernatant containing sheets of epithelial cells was discarded. The remaining tissues were rinsed twice with HBSS and digested in an appropriate amount of HBSS containing $0.15 \mathrm{mg} / \mathrm{mL}$ collagenase I (Invitrogen) at $37^{\circ} \mathrm{C}$ for $30 \mathrm{~min}$. The mixture was vigorously shaken every $10 \mathrm{~min}$ until the supernatant became turbid with dispersed stromal cells. The stromal cells were filtered through a $70 \mu \mathrm{m}$ nylon filter. Then, they were centrifuged, and the supernatant was removed by using HBSS to wash stromal cells twice. The cells were resuspended in a complete medium consisting of Dulbecco's modified Eagle's medium-nutrient mixture F-12 Ham (DMEM-F12, Sigma) with 20\% charcoal-stripped foetal bovine serum.

Cells were plated into 6 -well cell culture plates at a density of $2 \times 10^{5}$ cells per plate. Fresh culture medium containing $10 \mathrm{nmol} / \mathrm{L}$ oestradiol $-17 \beta\left(\mathrm{E}_{2}\right)$ and $1 \mu \mathrm{mol} / \mathrm{L}$ progesterone $\left(\mathrm{P}_{4}\right)$ were added in each well to artificially induce decidualisation.

\section{Digital gene expression profiling}

Ovariectomised mice were treated with subcutaneous injections of corn oil $\left(0.1 \mathrm{~mL} /\right.$ mouse, control), $E_{2}$ (100 ng/ mouse) and $\mathrm{P}_{4}(2 \mathrm{mg} /$ mouse) to establish the steroid hormone processing model. The mice were killed, and the uteri were collected at $24 \mathrm{~h}$ after each treatment. The tissues were sent to the Beijing Genomics Institute (BGl) and Digital gene expression profiling was performed by BGI Technology. 


\section{The inhibition and overexpression of Pdcd4 in ESCs (cell transfection)}

Seed cells were $70-90 \%$ confluent under normal growth conditions (typically $37^{\circ} \mathrm{C}$ and $5 \% \mathrm{CO}_{2}$ ) before transfection. The siRNA specifically targeting mouse Pdcd4, (siRNA1496/843/1096, $50 \mathrm{nmol} / \mathrm{L}$, GenePharma, China), an overexpression vector of Pdcd4, pCMV6-Pdcd4 and pCMV6vector $(515 \mathrm{ng} / \mu \mathrm{L}$ and $603 \mathrm{ng} / \mu \mathrm{L}$, Origene, Rockville, MD, USA) were mixed with Lipofectamine 3000 Reagent in appropriate proportions. The mixtures were incubated at room temperature for 5-10 min to allow complex formation. The DNA/siRNAlipid complexes were added dropwise onto cells. The plates were then gently swirled to uniformly distribute the transfection complexes among all cells. The cells were incubated at $37^{\circ} \mathrm{C}$ and $5 \% \mathrm{CO}_{2}$ and kept under observation. If cytotoxicity was observed after $6-8 \mathrm{~h}$, the complexes were removed, and fresh culture medium was added to the cells.

To investigate whether the down/upregulation of PDCD4 affects the establishment or maintenance of decidua function, we established several groups as follows: the ESCs were treated with siRNA-1096/pCMV6-Pdcd4 and then treated with $E_{2}$ and $P_{4}$ to analyse the effects of down/upregulation of PDCD4 on the establishment of decidualisation by measuring Dtprp mRNA (siRNA-1096/pCMV6-Pdcd4+ID, ID followed by transfection). Similarly, decidualisation was induced in ESCs, and the cells were treated with siRNA-1096/pCMV6$\mathrm{Pdcd} 4$ to explore the effects on the maintenance of decidua (ID+siRNA-1096/pCMV6-Pdcd4, transfection followed by ID). Cells without any treatment were depicted as Con.

\section{Flow cytometry for apoptosis analysis}

ESCs transfected with pCMV6-Pdcd4 and the negative control were harvested and incubated with Annexin $\mathrm{V}$ and $\mathrm{PI}$ at room temperature for $15 \mathrm{~min}$ in the dark and then quantitatively analysed using a FACS Vantage SE flow cytometer (BD Biosciences, Franklin Lakes, NJ, USA) (Liu et al. 2013).

\section{Statistical analysis}

Each experiment was repeated at least 3 times in each group. The collected data were analysed using SPSS, ver. 16.0 software (SPSS). Student's $t$-tests were performed to compare data in experiments having only two groups, while the data from experiments with multiple groups were analysed by one-way ANOVA (Students-Newman-Keuls). $P<0.05$ was considered significant.

\section{Results}

\section{Expression of Pdcd4 in mouse endometrium during early pregnancy}

To investigate the role of $\mathrm{Pdcd} 4$ in mouse endometrium, we examined the $P d c d 4 \mathrm{mRNA}$ and protein levels in the endometrium by qPCR and WB. The results indicated that the $P d c d 4$ mRNA and protein levels were increased as the days of pregnancy increased and were reduced significantly at IS from D5. Furthermore, the Pdcd4
mRNA and protein levels at IS were substantially lower than those at interimplantation sites (IIS) on D6 and D7 (Fig. 1A, B and C). ISH was performed to detect the localisation of Pdcd4 mRNA in the endometrium, and the results indicated that $P d c d 4$ was primarily located in the cytoplasm of luminal epithelium (LE) and glandular epithelium (GE) on D1, while positive signals were observed in the cytoplasm of ESCs from D4 (Fig. 1D). The expression pattern of PDCD4 protein detected by IHC was similar to that of the mRNA, but the protein was located in both the nuclei and cytoplasm (Fig. 1E).

\section{Expression of Pdcd4 in mouse endometrium during pseudopregnancy}

To explore whether the changes of $\mathrm{Pdcd} 4$ expression in mouse endometrium were induced by a blastocyst signal, we examined $\mathrm{Pdcd} 4$ levels in the endometrium of pseudopregnant mice. There were no significant differences in $\mathrm{Pdcd} 4$ gene expression found among PD1-PD7 (Fig. 2A, B and C). Pdcd4 was predominantly observed in the LE and GE (Fig. 2D). These results indicated that the differential expression of $\mathrm{Pdcd} 4$ in mouse endometrium was triggered by a blastocyst signal.

\section{Expression of Pdcd4 in mouse endometrium after artificially induced decidualisation in vivo and in vitro}

To further explore the association between Pdcd4 and decidualisation of the endometrium, we established artificially induced decidualisation models in vivo and in vitro. As shown in Fig. $3 \mathrm{~A}$ and $\mathrm{B}$, uterine swelling occurred after injection of corn oil, and the mass increased approximately 20 times. Both in vivo and in vitro, the expression levels of $\mathrm{Pdcd} 4$ were significantly decreased in response to artificially induced decidualisation (Fig. 3C, D, E and F). In addition, we also investigated the effects of oestrogen and progesterone on PDCD4 using digital gene expression profiling since they are key regulators during pregnancy. The results showed that oestrogen had an effect on PDCD4 (Table 2).

\section{Downregulation of Pdcd4 promotes decidualisation of mouse ESCS}

The ESCs of PD4 mice were used to confirm the effect of $\mathrm{Pdcd} 4$ on endometrial decidualisation. Figure 4A shows the identification of ESCs by immunofluorescence assays. The ESCs were transfected with siRNA and harvested $48 \mathrm{~h}$ later to detect the $\mathrm{Pdcd} 4 \mathrm{mRNA}$ and protein levels. The siRNA segment that had the maximum silencing effect (siRNA-1096) was chosen from the three segments (siRNA-1496, 843, 1096) for the next experiment (Fig. 4B, C and E). The Cleaved Caspase3, Caspase3 and $\mathrm{BAX} / \mathrm{BCL} 2$ proteins were detected by WB. Cell apoptosis was inhibited after downregulation of $\mathrm{Pdcd} 4$ (Fig. 4D and E). Figure 4F shows that the Dtprp levels 
A

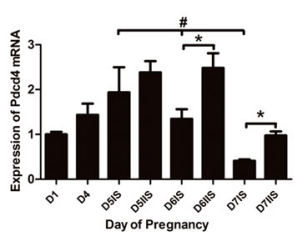

D
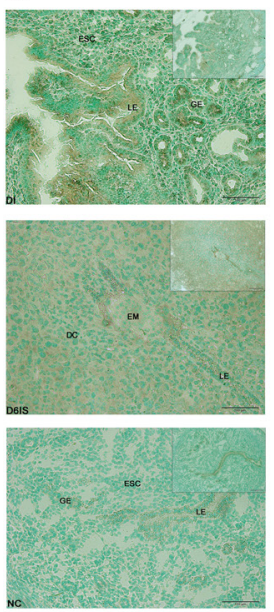

E
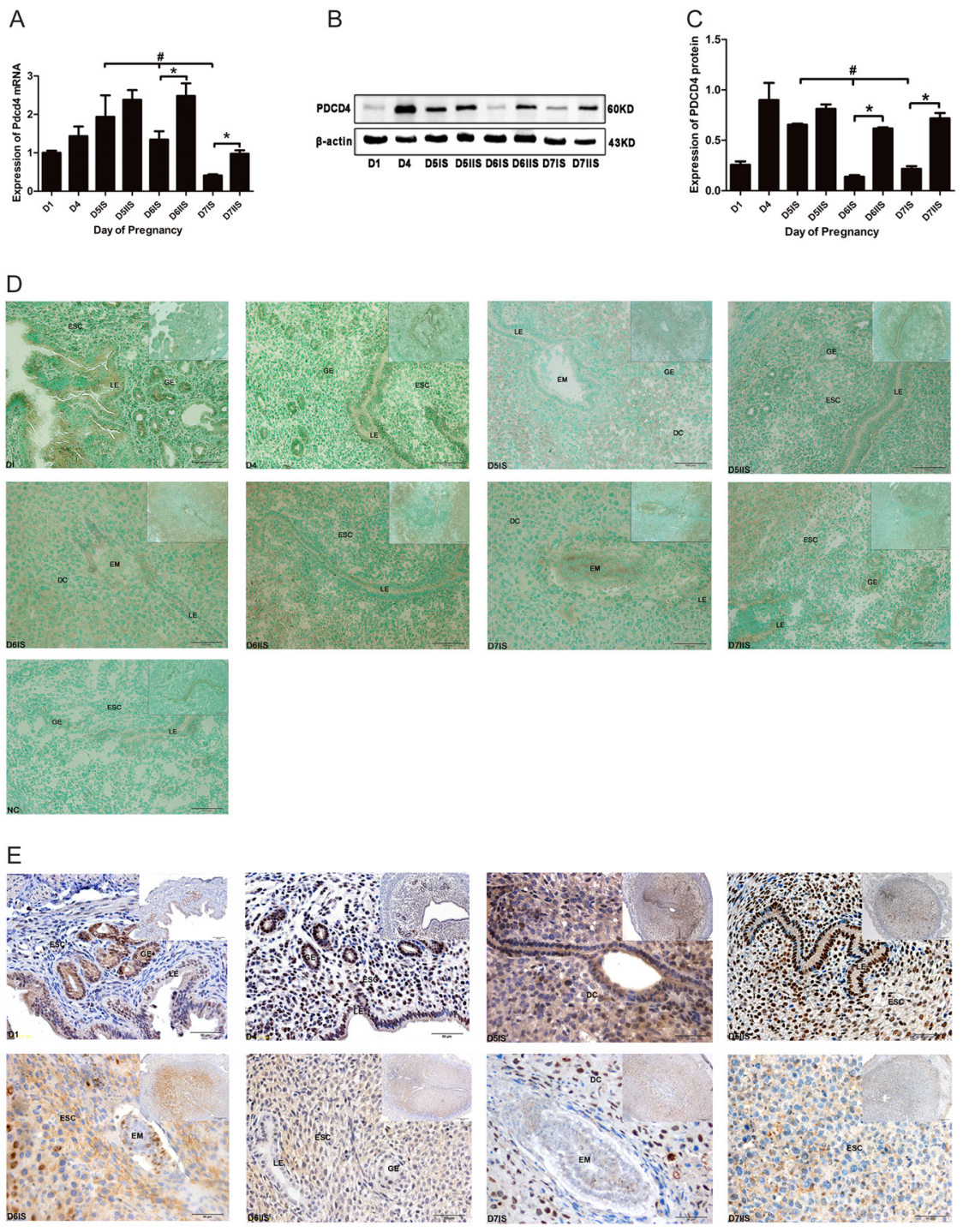

of the ID group were significantly higher than those in the control group, which indicated that the artificially induced decidualisation model in vitro was established successfully. Simultaneous comparison showed that the Dtprp mRNA level of the ID+siRNA-1096 (transfection followed by ID) group was significantly higher than

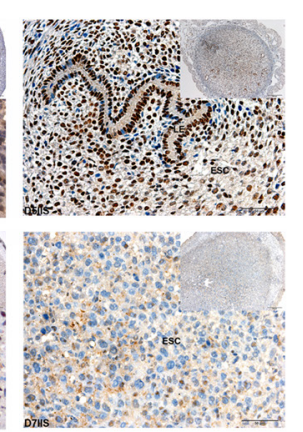

Figure 1 Expression of $P d c d 4$ in mouse endometrium during early pregnancy. (A) The relative mRNA expression of $P d c d 4$ in mouse endometrium. (B) Western blotting analysis of PDCD4 in mouse endometrium. $\beta$-Actin was used as a control. (C) Quantitation of PDCD4 protein expression by Western blotting. (D) The localisation of Pdcd4 mRNA was determined using ISH; the yellowish-brown stain indicates a positive signal. (E) The localisation of PDCD4 protein was determined by $\mathrm{IHC}$; the yellowish-brown stain indicates a positive signal. DC, decidualised cells; EM, embryo; ESC, endometrial stromal cells; GE, glandular epithelium; IS, implantation sites; IIS, interimplantation sites; LE, luminal epithelium; "indicates compared with D5IS; * and *indicate $P<0.05 ; * *$ indicates $P<0.01$.

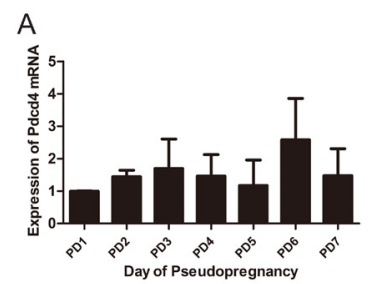

D

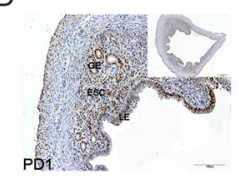

B

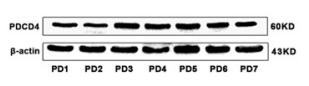

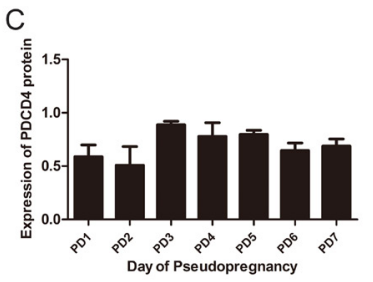

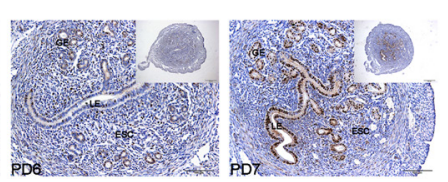

Figure 2 Expression of $\mathrm{Pdcd} 4$ in mouse endometrium during early pseudopregnancy. (A) The relative mRNA expression of $\mathrm{Pdcd} 4$ in mouse endometrium. (B) Western blotting analysis of PDCD4 protein in mouse endometrium. (C) Quantitation of PDCD4 protein expression by Western blotting. (D) The localisation of PDCD4 protein was determined by IHC; the yellowish-brown stain indicates a positive signal. ESC, endometrial stromal cells; GE, glandular epithelium; LE, luminal epithelium. 
A

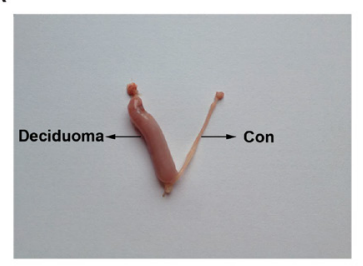

D

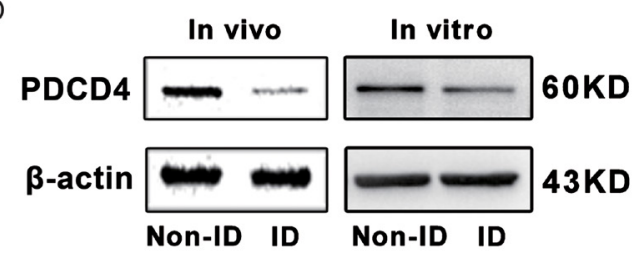

C

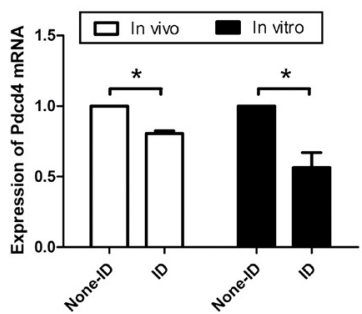

E

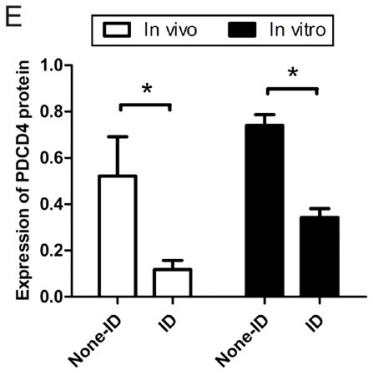

Figure 3 Expression of $P d c d 4$ in mouse endometrium during artificially induced decidualisation in vivo and in vitro. (A) Uterus from one representative mouse. The image shows the horn that received a physical stimulus (oil injection) to induce deciduoma formation on the left and the right as the internal control, which did not receive an oil injection. (B) The masses of the deciduoma and control uterine horns. (C) The level of $\mathrm{Pdcd} 4$ mRNA in mouse endometrium. (D) Western blotting analysis of PDCD4 protein in mouse endometrium. (E) Quantitation of PDCD4 protein expression by Western blotting. (F) The localisation of PDCD4 protein was determined by $\mathrm{IHC}$; the yellowish-brown stain indicates a positive signal. DC, decidualised cells; ESC, endometrial stromal cells; GE, glandular epithelium; LE, luminal epithelium. *Indicates $P<0.05,{ }^{* *}$ indicates $P<0.01$.

\section{PDCD4 expression in human endometrium}

The PDCD4 protein levels in different types of human endometrial samples during spontaneous abortion (control group, threatened-abortion group, missedabortion group) were detected. We found that the PDCD4 protein levels in the endometrium of the threatened-abortion and missed-abortion groups were substantially higher than those in the control group (Fig. 5A and B).

\section{Overexpression of Pdcd4 inhibits decidualisation of ESCs from mouse endometrium}

Since PDCD4 protein levels in human endometrial samples from the threatened-abortion and missedabortion groups were significantly higher than those in endometrial samples from the control group, we investigated the effects of $\mathrm{Pdcd} 4$ overexpression in mouse ESCs. Figure $6 \mathrm{~A}, \mathrm{~B}$ and $\mathrm{C}$ reveal that the ESCs transfected with pCMV6-Pdcd4 had a significant increase in $\mathrm{Pdcd} 4$ mRNA and PDCD4 protein levels compared to those in the control and negative control (pCMV6-vector) groups. The results of our flow cytometric experiments coupled with the upregulated levels of Cleaved Caspase3, Caspase 3 and BAX/BCL2 proteins indicated that $\mathrm{PdCd} 4$ overexpression increases apoptosis in ESCs (Fig. 6B, C, $D, E$ and F). Furthermore, the results of our experiments involving artificially induced decidualisation indicated that overexpression of the $P d c d 4$ gene could inhibit the establishment of decidualisation (pCMV6-Pdcd4+ID, ID followed by transfection). For the maintenance of the decidua (ID+pCMV6-Pdcd4, transfection followed by ID), a decrease in the expression levels of Dtprp was observed, but this trend was not statistically significant (Fig. 6G and $\mathrm{H}$ ).

\section{Discussion}

Successful embryo implantation is a fundamental step in the overall process of pregnancy. For implantation, not only does the conceived embryo need to become an active blastocyst, but this step must also synchronise with endometrial receptivity. The decidual cells are special tissues around the implantation site, which is composed of a variety of cellular components, and the establishment of decidualisation is regulated by hormones as well as the expression of various genes. This process plays a critical role in the maintenance of the early pregnancy (Pijnenborg 2002, Wang \& Dey 2006, Ramathal et al. 2010, Zhang et al. 2013, 2015).

Table 2 The results of digital gene expression profiling.

\begin{tabular}{lccc}
\hline Genes & Control & Oestrogen & Progesterone \\
\hline$P d c d 4$ & 331 & $91^{* *}$ & 301 \\
\hline
\end{tabular}

**Indicates compared with control and $P<0.01$. 


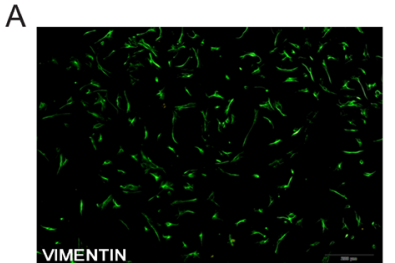

B

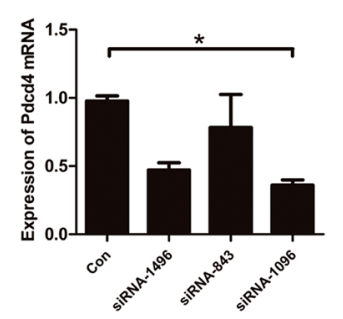

C

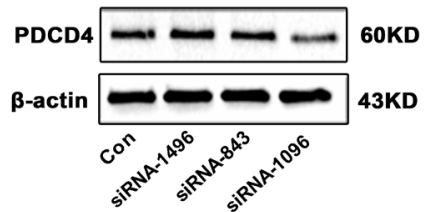

E

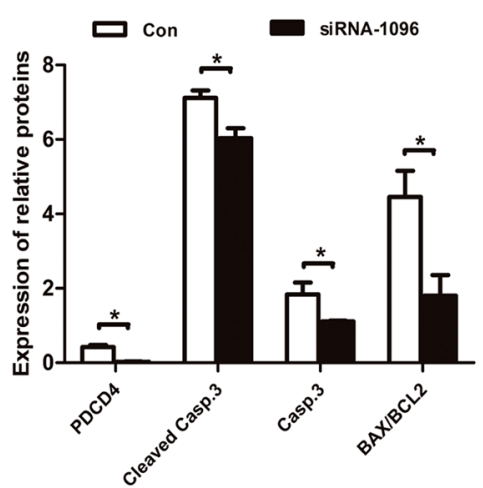

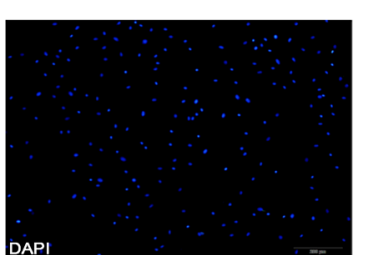

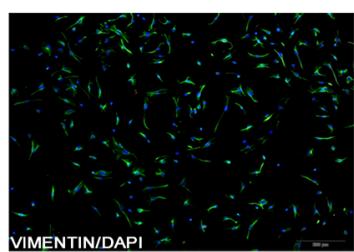

D

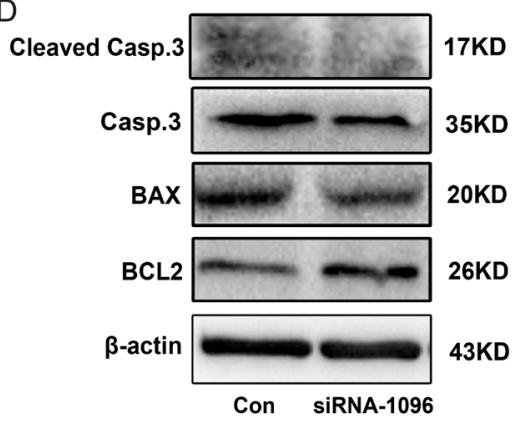

$\mathrm{F}$

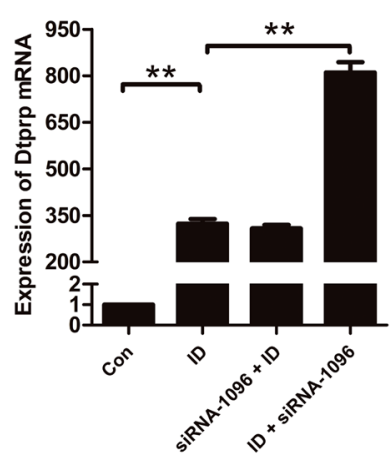

Figure 4 Effect of downregulated $\mathrm{Pdcd} 4$ on decidualisation of stromal cells. (A) Immunofluorescence analysis of VIMENTIN protein in ESCs. Green fluorescence indicates a positive signal. Scale bar $=200 \mu \mathrm{m}$. (B) The relative mRNA expression of $P d c d 4$ in ESCs after transfection with siRNA; siRNA-1096 showed the maximum silencing effect and was chosen for the next experiment. (C) Western blotting analysis of PDCD4 protein in ESCs after transfection with siRNA; siRNA-1096 showed the maximum silencing effect and was chosen for the next experiment. (D) Western blotting analysis of Cleaved Caspase3, Caspase3, BAX and BCL2 proteins in ESCs after transfection with siRNA-1096. (E) Quantitation of relative protein expression by Western blotting. (F) The relative mRNA expression of Dtprp in different treatment groups and Con. *Indicates $P<0.05$, **indicates $P<0.01$.
During the early pregnancy of mice, D4-D7 of pregnancy was the key period of the embryo implantation (Wang \& Dey 2006, Li et al. 2007, Ramathal et al.2010). The attachment reaction occurs on D4, when the uterus becomes receptive to implantation. Then, epithelial cells around the blastocyst undergo apoptosis, which causes the epithelium around that area to disintegrate to allow blastocyst invasion and implantation. While this occurs, the stromal cells near the blastocyst begin to proliferate and differentiate into decidual cells (Carson 2000, Boeddeker \& Hess 2015). We found that the Pdcd4 levels increased up to D4 to allow epithelial disintegration, which is necessary for embryo implantation. In addition, we found that the $\mathrm{Pdcd} 4 \mathrm{mRNA}$ and protein levels in the endometrium of IS on D5 were significantly higher than those at IS on D6 and D7 and that Pdcd4 gene expression was primarily observed in the decidual area. These results indicate that after embryo implantation, as the establishment and development of the decidua progresses, Pdcd4 expression is reduced to allow continued maintenance of decidua. These findings are consistent with the results of our in vivo and in vitro experiments involving artificially induced decidualisation before or after transfection with siRNA-1096. Furthermore, downregulation of $\mathrm{Pdcd} 4$ expression had little effect on the proliferation and differentiation of stromal cells but was important
A

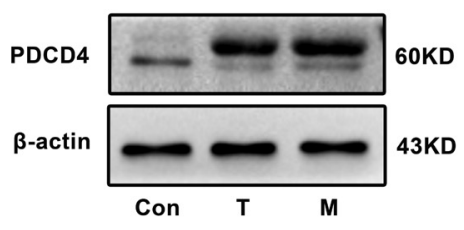

B

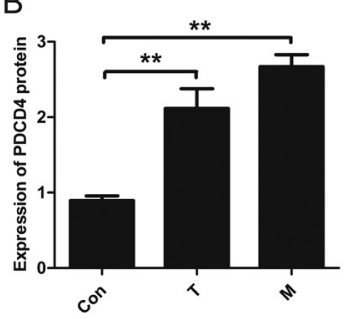

Figure 5 The expression of PDCD4 protein in human endometrium during spontaneous abortion (Con, control group; $\mathrm{T}$, threatenedabortion; $M$, missed-abortion). (A) Western blotting analysis of PDCD4 protein in the Con, $\mathrm{T}$, and M groups. (B) Quantitation of PDCD4 protein expression by Western blotting. 
A

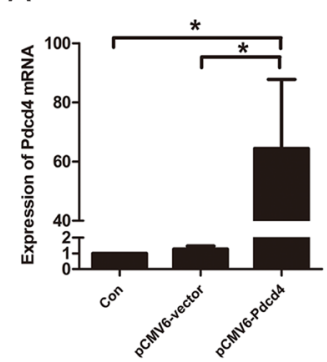

D

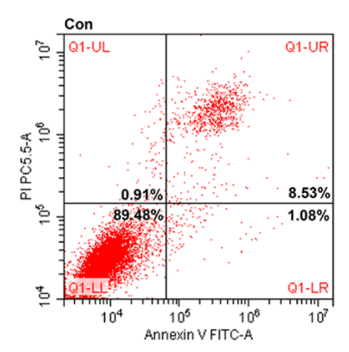

E
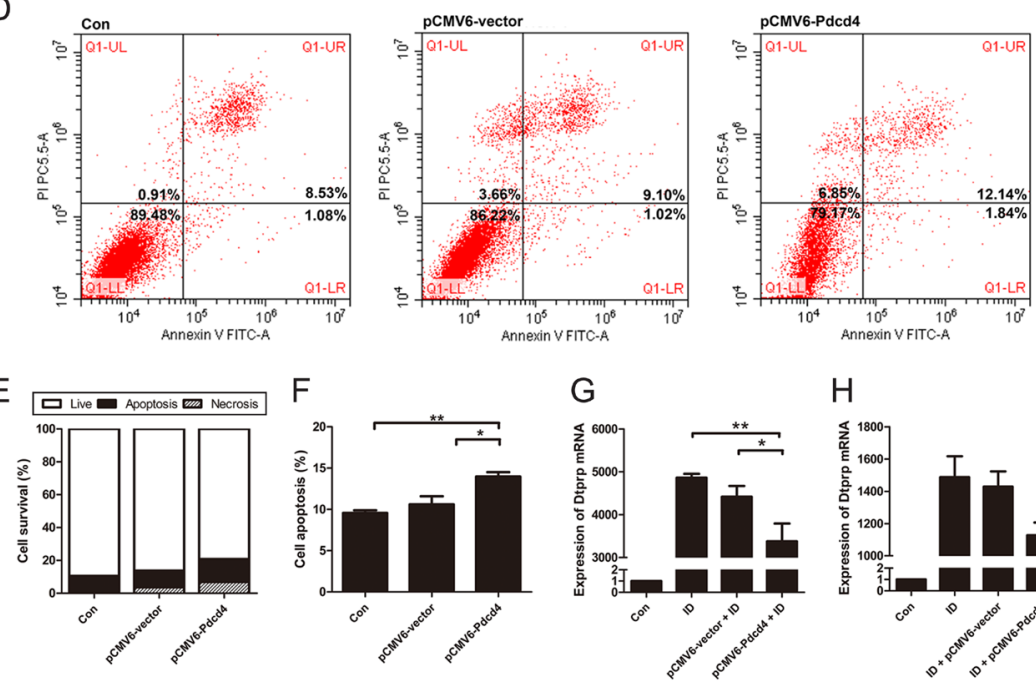

$\mathrm{H}$

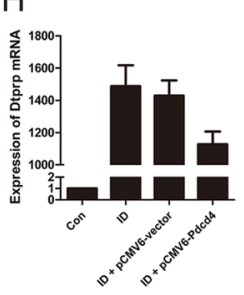

Figure 6 The effect of $P d c d 4$ overexpression on decidualisation of stromal cells. (A) The relative level of $P d c d 4$ mRNA in ESCs after transfection with the overexpression vector of Pdcd4. (B) Western blotting analysis of PDCD4, Cleaved Caspase3, Caspase3, BAX and $\mathrm{BCL} 2$ proteins in ESCs after transfection with the overexpression vector of $\mathrm{Pdcd} 4$. (C) Quantitation of relative protein expression by Western blotting. (D) The results of flow cytometry for apoptosis analysis of ESCs among the Con, pCMV6-vector and pCMV6$\mathrm{Pdcd} 4$ groups. (E) The data for cell survival presented in D. (F) The statistical analysis of the cell apoptosis rate presented in D. (G) The relative mRNA expression of Dtprp after treatment with pCMV6-vector/Pdcd4+ID. (H) The relative mRNA expression of Dtprp after treatment with ID+pCMV6-vector/Pdcd4.

*Indicates $P<0.05, * *$ indicates $P<0.01$. for the apoptosis of decidua. To investigate whether changes in Pdcd4 gene expression were induced by embryo signals rather than by hormonal regulation, we conducted experiments on pseudopregnant mice. The results clearly verified our hypothesis.

The normal low expression of $\mathrm{Pdcd} 4$ has favourable effects on decidualisation; however, what about abnormal expression? We observed that the protein levels of PDCD4 in the threatened-abortion and missedabortion groups were notably increased. Hence, we hypothesised that the high levels of PDCD4 could be a contributing factor to the occurrence of abortion. As PDCD4 protein increased in the overexpression model, apoptosis was enhanced accordingly. Meanwhile, $\mathrm{Pdcd} 4$ overexpression had adverse effects on the establishment of decidualisation. However, for the maintenance of decidua function, we observed a downward trend of Dtprp mRNA level, but this result was not significant. We attributed this to the survival time of stromal cells, which is only 6-7 days in vitro. After artificially induced decidualisation, the activity and function of stromal cells were weakened, and it was difficult to achieve an effective transfection. These present results further illustrated the detrimental effects of high levels of PDCD4 on decidualisation and hence on pregnancy.

Data from recent publications, as well as our results from this study, indicate that apoptosis plays a major role in the female reproductive tract. Apoptosis of
ESCs can be initiated via an intrinsic or mitochondriaassociated pathway. The aggregation of cytochrome C activates Caspase9 and consequently Caspase3 to induce apoptosis (Joswig et al. 2003). Our results demonstrated that overexpression of the $\mathrm{Pdcd} 4$ gene increases apoptosis in ESCs via the mitochondriaassociated pathway. Several studies reported that nondifferentiated cells are resistant to apoptosis - even after exposure to death receptors - because these cells have the potential to proliferate and differentiate. Accordingly, after artificially induced decidualisation, differentiated ESCs showed high sensitivity to Caspase3 activation (Boeddeker \& Hess 2015). This phenomenon probably occurs because the decidual cells surrounding the embryo have higher expression of pro-apoptotic proteins than the adjacent stromal cells, which express anti-apoptotic BCL2 proteins instead. Collectively, neither a premature nor delayed end of decidualisation can result in a normal pregnancy. Although proliferation and differentiation of stromal cells are very important for the formation of the decidua, timely apoptosis is equally important for this process (Mikhailov 2003).

In summary, appropriate expression of the Pdcd4 gene in mouse endometrium plays an important role in the establishment and maintenance of decidua function. Thus, we hypothesised that $\mathrm{Pdcd} 4$ gene expression affects embryo implantation. Abnormal expression of the $\mathrm{Pdcd} 4$ gene in the endometrium is 
likely to be detrimental to pregnancy. These results could help develop novel strategies or new therapeutic targets for treating disorders of the reproductive system, including infertility.

\section{Declaration of interest}

The authors report that no conflict of interest exists to prejudice the impartiality of the research reported.

\section{Funding}

This work was supported by the Natural Science Foundation Project of CQ CSTC (Nos. cstc2017jcyjAX0287).

\section{Acknowledgements}

The authors would like to express gratitude to all members in their research group for their technical support.

\section{References}

Avi Ashkenazi VMD 1998 Death receptor: signaling and modulation. Science 281 1305-1308. (https://doi.org/10.1126/science.281.5381.1305)

Bigsby RM \& Li A 1994 Differentially regulated immediate early genes in the rat uterus. Endocrinology 134 1820-1826. (https://doi.org/10.1210/ endo.134.4.8137748)

Boeddeker SJ \& Hess AP 2015 The role of apoptosis in human embryo implantation. Journal of Reproductive Immunology 108 114-122. (https://doi.org/10.1016/j.jri.2015.02.002)

Bowen JA \& Burghardt RC 2000 Cellular mechanisms of implantation in domestic farm animals. Seminars in Cell and Developmental Biology 11 93-104. (https://doi.org/10.1006/scdb.2000.0155)

Carson DD, Bagchi I, Dey SK, Enders AC, Fazleabas AT, Lessey BA \& Yoshinaga K 2000 Embryo implantation. Developmental Biology 223 217-237. (https://doi.org/10.1006/dbio.2000.9767)

Das SK, Das N, Wang J, Lim H, Schryver B, Plowman GD \& Dey SK 1997 Expression of betacellulin and epiregulin genes in the mouse uterus temporally by the blastocyst solely at the site of its apposition is coincident with the "window" of implantation. Developmental Biology 190 178-190. (https://doi.org/10.1006/dbio.1997.8694)

Dey SK, Lim H, Das SK, Reese J, Paria BC, Daikoku T \& Wang H 2004 Molecular cues to implantation. Endocrine Reviews 25 341-373. (https:// doi.org/10.1210/er.2003-0020)

Ding YB, He JL, Liu XQ, Chen XM, Long CL \& Wang YX 2012 Expression of DNA methyltransferases in the mouse uterus during early pregnancy and susceptibility to dietary folate deficiency. Reproduction 144 91-100. (https://doi.org/10.1530/REP-12-0006)

Ding L, Gao F, Zhang M, Yan W, Tang R, Zhang C \& Chen ZJ 2016 Higher PDCD4 expression is associated with obesity, insulin resistance, lipid metabolism disorders, and granulosa cell apoptosis in polycystic ovary syndrome. Fertility and Sterility 105 1330.e3-1337.e3. (https://doi. org/10.1016/j.fertnstert.2016.01.020)

Everett LM, Li A, Devaraju G, Caperell-Grant A \& Bigsby RM 1997 A novel estrogen-enhanced transcript identified in the rat uterus by differential display. Endocrinology 138 3836-3841. (https://doi.org/10.1210/ endo.138.9.5384)

Green DR \& Reed JC 1998 Mitochondria and apoptosis. Science 281 1309-1312. (https://doi.org/10.1126/science.281.5381.1309)

Huet-Hudson YM, Chakraborty C, De SK, Suzuki Y, Andrews GK \& Dey SK 1990 Estrogen regulates the synthesis of epidermal growth factor in mouse uterine epithelial cells. Molecular Endocrinology 4 510-523. (https://doi.org/10.1210/mend-4-3-510)

Joswig A, Gabriel HD, Kibschull M \& Winterhager E 2003 Apoptosis in uterine epithelium and decidua in response to implantation: evidence for two different pathways. Reproductive Biology and Endocrinology 1 44. (https://doi.org/10.1186/1477-7827-1-44)

Kang MJ, Ahn HS, Lee JY, Matsuhashi S \& Park WY 2002 Up-regulation of PDCD4 in senescent human diploid fibroblasts. Biochemical and Biophysical Research Communications 293 617-621. (https://doi. org/10.1016/S0006-291X(02)00264-4)

Lankat-Buttgereit B \& Goke R 2009 The tumour suppressor Pdcd4: recent advances in the elucidation of function and regulation. Biology of the Cell 101 309-317. (https://doi.org/10.1042/BC20080191)

Larsen EC, Christiansen OB, Kolte AM \& Macklon N 2013 New insights into mechanisms behind miscarriage. BMC Medicine 11 154. (https:// doi.org/10.1186/1741-7015-11-154)

Li Q, Kannan A, Wang W, Demayo FJ, Taylor RN, Bagchi MK \& Bagchi IC 2007 Bone morphogenetic protein 2 functions via a conserved signaling pathway involving Wnt4 to regulate uterine decidualization in the mouse and the human. Journal of Biological Chemistry 282 31725-31732. (https://doi.org/10.1074/jbc.M704723200)

Liang XH, Zhao ZA, Deng WB, Tian Z, Lei W, Xu X, Zhang XH, Su RW \& Yang ZM 2010 Estrogen regulates amiloride-binding protein 1 through CCAAT/enhancer-binding protein-beta in mouse uterus during embryo implantation and decidualization. Endocrinology 151 5007-5016. (https://doi.org/10.1210/en.2010-0170)

Liu X, Gao R, Chen X, Zhang H, Zheng A, Yang D, Ding Y, Wang Y \& He J 2013 Possible roles of mmu-miR-141 in the endometrium of mice in early pregnancy following embryo implantation. PLOS ONE 8 e67382. (https://doi.org/10.1371/journal.pone.0067382)

Long X, Zhang M, Chen X, He J, Ding Y, Zhang C, Liu X \& Wang Y 2015 Expression of KRAS in the endometrium of early pregnant mice and its effect during embryo implantation. Reproductive BioMedicine Online 31 51-61. (https://doi.org/10.1016/j.rbmo.2015.04.005)

Messinis IE, Messini CI, Daponte A, Garas A \& Mahmood T 2016 The current situation of infertility services provision in Europe. European Journal of Obstetrics and Gynecology and Reproductive Biology 207 200-204. (https://doi.org/10.1016/j.ejogrb.2016.10.004)

Mikhailov VM 2003 Life cycle of decidual cells. International Review of Cytology 227 1-63. (https://doi.org/10.1016/S0074-7696(03)01007-6)

Mudduluru G, Medved F, Grobholz R, Jost C, Gruber A, Leupold JH, Post S, Jansen A, Colburn NH \& Allgayer H 2007 Loss of programmed cell death 4 expression marks adenoma-carcinoma transition, correlates inversely with phosphorylated protein kinase $B$, and is an independent prognostic factor in resected colorectal cancer. Cancer 110 1697-1707. (https://doi.org/10.1002/cncr.22983)

Ni H, Sun T, Ding NZ, Ma XH \& Yang Z-M 2002 Differential expression of microsomal prostaglandin $\mathrm{E}$ synthase at implantation sites and in decidual cells of mouse uterus. Biology of Reproduction 67 351-358. (https://doi.org/10.1095/biolreprod67.1.351)

Osteen KG, Hill GA, Hargrove JT \& Gorstein F 1989 Development of a method to isolate and culture highly purified populations of stromal and epithelial cells from human endometrial biopsy specimens. Fertility and Sterility 52 965-972. (https://doi.org/10.1016/S0015-0282(16)53160-4)

Ozpolat B, Akar U, Steiner M, Zorrilla-Calancha I, Tirado-Gomez M, Colburn N, Danilenko M, Kornblau S \& Berestein GL 2007 Programmed cell death-4 tumor suppressor protein contributes to retinoic acidinduced terminal granulocytic differentiation of human myeloid leukemia cells. Molecular Cancer Research 5 95-108. (https://doi. org/10.1158/1541-7786.MCR-06-0125)

Paria BC, Huet-Hudson YM \& Dey SK 1993 Blastocyst's state of activity determines the "window" of implantation in the receptive mouse uterus. Developmental Biology 90 10159-10162.

Pijnenborg R 2002 Implantation and immunology: maternal inflammatory and immune cellular responses to implantation and trophoblast invasion. Reproductive BioMedicine Online 4 (Supplement 3) 14-17. (https://doi. org/10.1016/S1472-6483(12)60110-2)

Ramathal CY, Bagchi IC, Taylor RN \& Bagchi MK 2010 Endometrial decidualization: of mice and men. Seminars in Reproductive Medicine 28 17-26. (https://doi.org/10.1055/s-0029-1242989)

Wang H \& Dey SK 2006 Roadmap to embryo implantation: clues from mouse models. Nature Reviews Genetics 7 185-199. (https://doi. org/10.1038/nrg1808)

Wang YX, Zhao JR, Xu YY, Wu WB \& Zhang HJ 2017 miR-21 is overexpressed in hydatidiform mole tissues and promotes proliferation, migration, and invasion in choriocarcinoma cells. International Journal 
of Gynecological Cancer 27 364-374. (https://doi.org/10.1097/ IGC.0000000000000861)

Zhang S, Lin H, Kong S, Wang S, Wang H, Wang H \& Armant DR 2013 Physiological and molecular determinants of embryo implantation. Molecular Aspects of Medicine 34 939-980. (https://doi.org/10.1016/j. mam.2012.12.011)

Zhang Y, Goodfellow R, Li Y, Yang S, Winters CJ, Thiel KW, Leslie KK \& Yang B 2015 NEDD4 ubiquitin ligase is a putative oncogene in endometrial cancer that activates IGF-1R/PI3K/Akt signaling. Gynecologic Oncology 139 127-133. (https://doi.org/10.1016/j.ygyno.2015.07.098)

Zhang X, Liu R, Huang B, Zhang X, Yu W, Bao C, Li J \& Sun C 2016 Programmed cell death 4 and BCR-ABL fusion gene expression are negatively correlated in chronic myeloid leukemia. Oncology Letters 12 2976-2981. (https://doi.org/10.3892/ol.2016.4942)

Received 14 July 2017

First decision 3 August 2017

Revised manuscript received 7 February 2018

Accepted 19 February 2018 